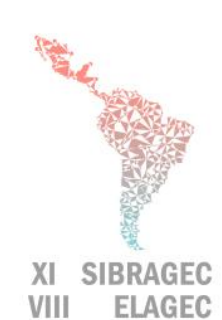

VIII ELAGEC

\section{SIMPÓSIO BRASILEIRO DE GESTÃO E ECONOMIA DA CONSTRUÇÃO \\ VIII ENCUENTRO LATINOAMERICANO DE GESTIÓN \\ Y ECONOMÍA DE LA CONSTRUCCIÓN}

Do conhecimento à ação: práticas avançadas de gestão da produção Londrina, Paraná, Brasil. 23 a 25 de outubro de 2019

\title{
A EVOLUÇÃO DO CONHECIMENTO DE CONSTRUTIBILIDADE
}

\author{
ABREU, João Paulo Maciel de; MARCHIORI, Fernanda Fernandes; OVIEDO \\ HAITO, Ricardo Juan José.
}

(1) UFSC, e-mail: joaopaulojpma@ @otmail.com (2) UFSC, e-mail: fernanda.marchiori@ufsc.br,

(3) UFSC, e-mail: mltca2@gmail.com

\begin{abstract}
In civil construction there is a constant search for improvement. Among the ways to achieve it, the concept of constructability stands out. Constructability can be understood as a characteristic of a building or construction project to be easily performed. Since 1962, in Emmerson Report, this concept is growing and involving different areas of construction research. Notwithstanding, there is no consensus about the meaning of this concept, that can be associated to terms such as constructability, buildability, among others, with similar constructs but different scopes in construction life-cycle. The aim of this paper is to show the evolution of constructability knowledge in researches conducted in Brazil and other countries, specially USA, UK, Singapore and Hong Kong. To reach this aim, a bibliometric integrative review was carried out in international databases, including journals and event proceedings, and Brazilian thesis repositories. Three hundred forty-three researches mentioned the terms "constructability" or "buildability" as well its equivalent "construtibilidade". As a result, it was observed the consolidation of this concept in 2000's, and the emergence of new ways and tools related to constructability analysis such as indicators systems or analysis using BIM 4D; contributing to the discussion about how to put the constructability concept into practice.
\end{abstract}

Keywords: Constructability. Buildability. Integrative Review.

\section{INTRODUÇÃO}

No ano de 1962, Sir. Harold Emmerson publicou o documento "Emmerson Report", em que aponta inconsistências entre o que era disposto no escopo do produto e a posterior execução de empreendimentos de construção civil. Para alguns autores, é apontado como um dos pioneiros em citar que a separação entre profissionais atuantes em projeto e construção não seria favorável à construtibilidade (sem citar esse termo explicitamente) (LAM; WONG; CHAN, 2006; KIFOKERIS; XENIDIS, 2017).

É constante a realização de novas pesquisas na área de construção, buscando sua melhoria em diversos aspectos, e um deles é a construtibilidade, que é uma característica que indica o grau de facilidade de execução de uma edificação. $O$ acréscimo de construtibilidade ocorre quando se insere em etapas iniciais (viabilidade e escopo do produto) todo o conhecimento existente até as operações construtivas (SABBATINI, 1989; ASCE, 1991).

Quando a etapa do empreendimento considerada é a de definição do design do produto, tem-se o que em língua inglesa é definido como buildability (JARKAS, 2010). Já a

ABREU, J.P.M.; MARCHIORI, F.F.; OVIEDO HAITO, R.J.J. A evolução do conhecimento de construtibilidade. In: SIMPÓSIO BRASILEIRO DE GESTÃO E ECONOMIA DA CONSTRUÇÃO, 11. 2019, Londrina. Anais [...]. Porto Alegre: ANTAC, 2019. Disponível em: https://www.antaceventos.net.br/index.php/sibragec/sibragec2019/paper/view/249 
SIBRAGEC - ELAGEC 2019 - de 23 a 25 de outubro - LONDRINA - PR

definição com desenvolvimento estadunidense chamada constructability envolve mais etapas do ciclo de vida do processo de construção no contexto de melhoria da construtibilidade (LAM; WONG; CHAN, 2006). Com o passar do tempo, algumas publicações internacionais citam casos de uso dos termos buildability e constructability como um mesmo conceito (LAM; WONG; CHAN, 2006), ou ainda que o termo constructability passasse a integrar aspectos da edificação em uso (LAM et al., 2008).

Considerando que a construtibilidade envolve o uso ótimo do conhecimento em construção existente advindo dos stakeholders do setor ou eventuais bancos de dados (TATUM, 2005), tem-se margem a interpretações subjetivas, apesar de ser relevante se valer de tal recurso. Então, uma forma objetiva de análise de construtibilidade está na criação e uso de sistemas de indicadores de construtibilidade. A concepção desses sistemas, formatos e objetivos é diversa, praticamente não havendo publicações nessa linha no Brasil. No exterior, destacam-se sistemas com foco no design do produto ou buildability, implantados como política pública em Singapura (POH; CHEN, 1998; CHIANG; CHAN; LOK, 2006) ou dentro de softwares de avaliação (KANNAN; SANTHI, 2018).

O objetivo dessa pesquisa foi de traçar a evolução do conhecimento existente sobre "construtibilidade", elucidando suas diferentes abordagens e ferramentas de implantação como filosofia de projeto. Para tanto, foram consideradas pesquisas desenvolvidas no Brasil e no exterior.

\section{MÉTODO}

O método utilizado foi a revisão bibliométrica integrativa (BOTELHO; CUNHA; MACEDO, 2011). Esse tipo de revisão permite sumarizar estudos primários e secundários e levantar o conhecimento pré-existente em determinado tema (Quadro1). Após, novos conhecimentos e discussões são gerados com a análise de resultados de pesquisa.

Uma primeira etapa, dentro do método adotado, consistiu em definir palavras-chave e caracteres de pesquisa (strings) a serem utilizados em bases de dados e repositórios. Para isso, fez-se revisão narrativa, que permite criar familiaridade com o tema a ser pesquisado e estabelecer critérios mais eficientes quando aplicado método sistematizado de revisão (BOTELHO; CUNHA; MACEDO, 2011). Após, realizou-se a revisão bibliométrica integrativa, com um método sistemático de seleção de publicações para composição de um portfólio de referências, seguindo as etapas descritas no Quadro 1. 
SIBRAGEC - ELAGEC 2019 - de 23 a 25 de outubro - LONDRINA - PR

Quadro 1a - Processo de Revisão Bibliométrica Integrativa

\begin{tabular}{|c|c|c|}
\hline & Etapa & Atividades \\
\hline 1 & $\begin{array}{l}\text { Identificação do tema e } \\
\text { seleção da questão de } \\
\text { pesquisa }\end{array}$ & $\begin{array}{l}\text { - Definição do problema. } \\
\text { - Formulação de uma pergunta de pesquisa - Como a construtibilidade } \\
\text { vem sendo conceituada e avaliada, nacional e internacionalmente? } \\
\text { - Definição da estratégia de busca - buscar produção nacional e } \\
\text { internacional, período de tempo aberto e sem filtros (diferentes ou } \\
\text { indisponíveis segundo bases distintas). } \\
\text { - Definição dos descritores - uso dos termos "constructability" e } \\
\text { "buildability" em inglês, e "construtibilidade" em português. } \\
\text { - Definição das bases de dados. }\end{array}$ \\
\hline 2 & $\begin{array}{l}\text { Estabelecimento dos } \\
\text { critérios de inclusão e } \\
\text { exclusão } \\
\end{array}$ & $\begin{array}{l}\text { - Uso das bases de dados. } \\
\text { - Busca dos estudos com base nos critérios de inclusão e exclusão. }\end{array}$ \\
\hline 3 & $\begin{array}{l}\text { Identificação dos estudos } \\
\text { pré-selecionados e } \\
\text { selecionados. }\end{array}$ & $\begin{array}{l}\text { - Leitura dos resumos, palavras-chave e títulos das publicações. } \\
\text { - Organização dos estudos pré-selecionados. } \\
\text { - Identificação dos estudos selecionados. }\end{array}$ \\
\hline 4 & $\begin{array}{l}\text { Categorização dos } \\
\text { estudos selecionados }\end{array}$ & $\begin{array}{l}\text { - Elaboração e uso da matriz de síntese. } \\
\text { - Categorização e análise de informações. } \\
\text { - Formação de uma biblioteca individual. } \\
\text { - Análise crítica dos estudos selecionados. }\end{array}$ \\
\hline
\end{tabular}

Fonte: Botelho, Cunha e Macedo (2011), os Autores (2019).

\section{Quadro 1b - Processo de Revisão Bibliométrica Integrativa}

\begin{tabular}{|c|c|c|}
\hline & Etapa & \multicolumn{1}{c|}{ Atividades } \\
\hline $\mathbf{5}$ & $\begin{array}{c}\text { Análise e interpretação } \\
\text { dos resultados. }\end{array}$ & $\bullet$ Discussão dos resultados. \\
\hline $\mathbf{6}$ & $\begin{array}{c}\text { Apresentação da } \\
\text { revisão/Síntese do } \\
\text { conhecimento. }\end{array}$ & $\begin{array}{c}\text { • Criação de um documento que descreva detalhadamente a revisão - } \\
\text { paper no SIBRAGEC. } \\
\text { • Propostas para estudos futuros. }\end{array}$ \\
\hline
\end{tabular}

Fonte: Botelho, Cunha e Macedo (2011), os Autores (2019).

Feitas essas etapas, obteve-se o portfólio de publicações disponível no seguinte sítio eletrônico: https://www.engjpma.com.br/2015/07/a-evolucao-do-conhecimento-em.htm 1. A Figura 1 ilustra o processo de seleção desde os resultados nas bases de dados até a obtenção do portfólio: 
SIBRAGEC - ELAGEC 2019 - de 23 a 25 de outubro - LONDRINA - PR

Figura 1 - Processo de seleção

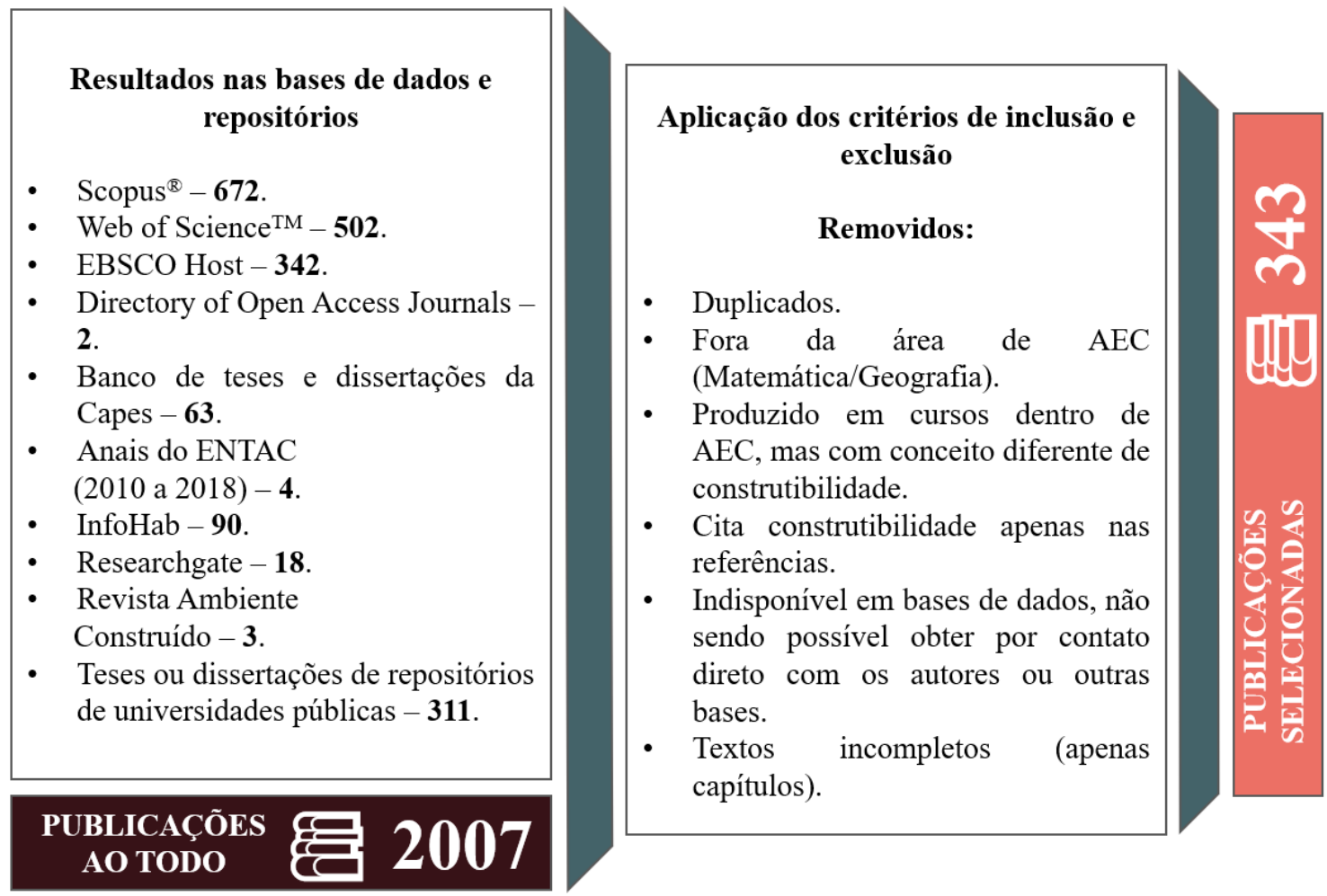

Fonte: os Autores (2019)

Algumas publicações foram filtradas nos ambientes virtuais das bases e repositórios, por meio da leitura de títulos e resumos. As demais, analisadas por qual a abordagem do termo "construtibilidade". Há outros entendimentos para construtibilidade que diferem do trabalhado nesse estudo, como nas áreas de Urbanismo, e Geografia, com construtibilidade sendo a capacidade ou não de edificar em dado terreno, ou ainda na área de Matemática, onde, por exemplo, Lima (2018) versa sobre construções geométricas utilizando software.

\section{RESULTADOS E DISCUSSÃO}

Dentre as diferentes abordagens sobre construtibilidade, a mais antiga é a de conceituação do termo, em publicações dos anos 1980 e 1990, menos presente após os anos 2000, como no estudo de Gerth et al. (2013). Durante esse período, no Brasil, há trabalhos pioneiros como as teses de Sabbatini (1989) e de Melhado (1994). Também há discussões em artigos de eventos como o ENTAC 1998.

A Figura 2 apresenta as categorias de estudos que mencionam "construtibilidade" ao longo de seus textos e que foram selecionados nessa revisão. A maioria desses estudos é de dissertações nacionais, nos últimos vinte anos, onde apenas há menção do termo como algo consolidado, entendido como a facilidade de construir e utilizado como adjetivo para métodos construtivos ou técnicas inovadoras. 
SIBRAGEC - ELAGEC 2019 - de 23 a 25 de outubro - LONDRINA - PR

Figura 2 - Abordagens sobre construtibilidade

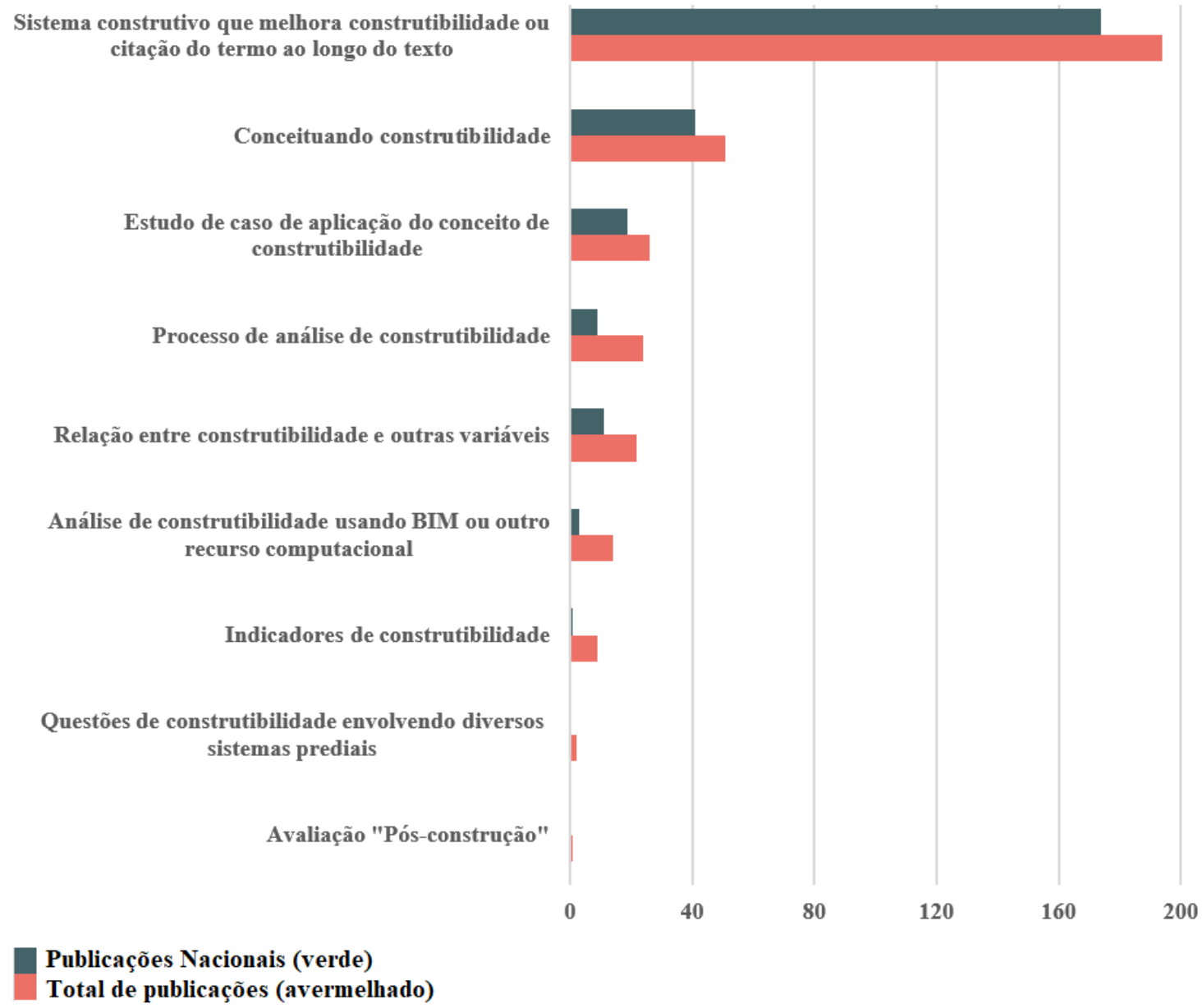

Fonte: os Autores (2019)

Ao analisar os maiores meios de publicação, visto que muitas das dissertações não agregam ao estudo de construtibilidade em si, mas a citam apenas, verificou-se a aderência à Lei de Bradford (1934) desconsiderando essa parcela de publicações, obtendo-se o disposto na Figura 3. Essa Lei da Bibliometria afirma a existência de zonas de produtividade bibliográfica, onde a faixa superior apresenta um menor número de autores/meios de publicação e maior produção bibliográfica, relação esta que se inverte nas faixas inferiores (MACHADO JÚNIOR et al, 2016; ARAÚJO, 2006). Observa-se que é possível seccionar em aproximadamente um terço das publicações cada faixa, com os maiores meios de publicação (no núcleo ou Faixa 1) sendo os anais do evento ENTAC (nacional) e o periódico Journal of Construction Engineering and Management (internacional). 
SIBRAGEC - ELAGEC 2019 - de 23 a 25 de outubro - LONDRINA - PR

Figura 3 - Zonas de produtividade da Lei de Bradford

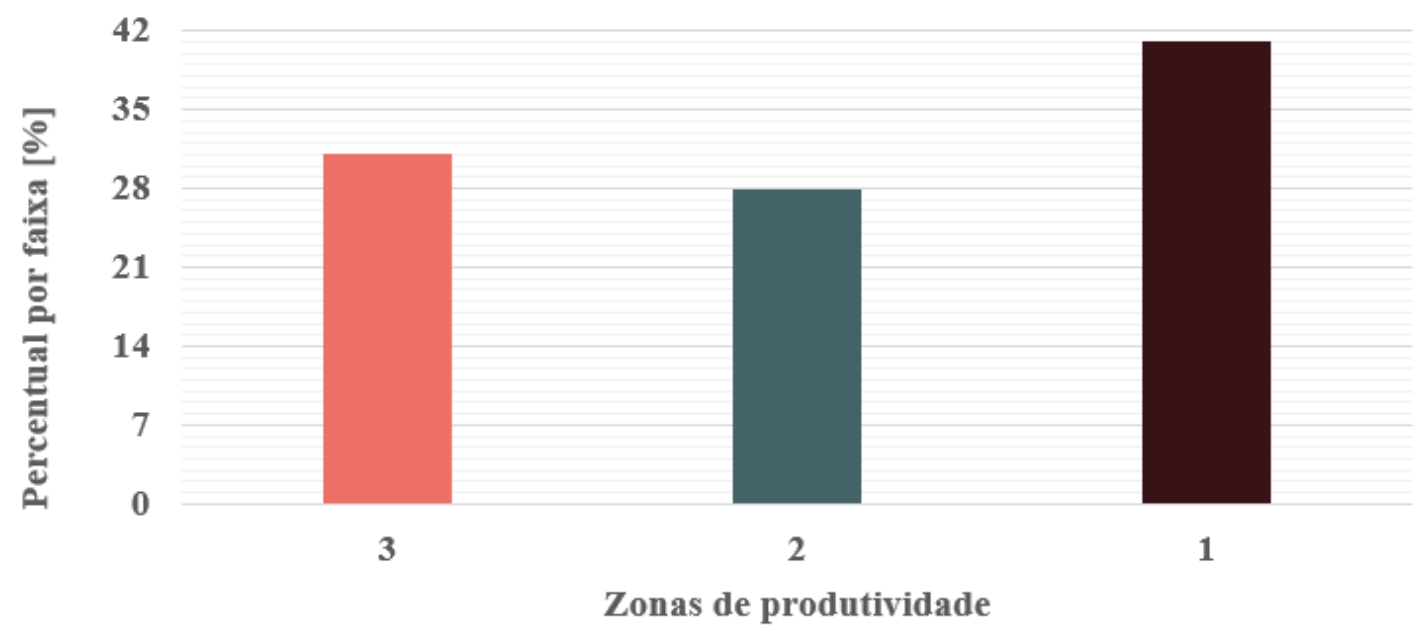

\begin{tabular}{|c|c|c|c|}
\hline \multirow{3}{*}{ Faixa 1: } & Meio de publicação & $\mathbf{N}^{\mathbf{0}}$ & \\
\hline & $\begin{array}{l}\text { Journal of Construction Engineering and } \\
\text { Management }\end{array}$ & 31 & $24 \%$ \\
\hline & ENTAC & 21 & $17 \%$ \\
\hline \multirow[t]{12}{*}{ Faixa 2: } & Journal of Architectural Engineering & 2 & $2 \%$ \\
\hline & International Journal for Housing Science & 2 & $2 \%$ \\
\hline & Building and Environment & 2 & $2 \%$ \\
\hline & Journal of Civil Engineering and Management & 2 & $2 \%$ \\
\hline & SIBRAGEC & 2 & $2 \%$ \\
\hline & $\begin{array}{l}\text { Workshop brasileiro de gestão do processo de } \\
\text { projeto na construção de edifícios }\end{array}$ & 2 & $2 \%$ \\
\hline & $\begin{array}{l}\text { International Seminar on Structural Masonry for } \\
\text { Developing Countries }\end{array}$ & 2 & $2 \%$ \\
\hline & Congresso Técnico-científico de Engenharia Civil & 2 & $2 \%$ \\
\hline & Revista Ambiente Construído & 3 & $2 \%$ \\
\hline & Automation in Construction & 5 & $4 \%$ \\
\hline & SBQP & 5 & $4 \%$ \\
\hline & Journal of Management in Engineering & 7 & $6 \%$ \\
\hline Faixa 3: & $\begin{array}{l}\text { Outros trinta e nove meios de publicação com um } \\
\text { texto }\end{array}$ & 39 & $31 \%$ \\
\hline
\end{tabular}

Fonte: os Autores (2019)

Alguns pesquisadores verificam em empresas de construção a incorporação de conceitos de construtibilidade (redução do número de partes, modularidade, padronização, dentre outros) dentro de suas rotinas, em estudos de caso como o de Chiang, Chan e Lok (2006). Ela pode ocorrer como um processo de análise de construtibilidade onde, com primeiras definições do produto e seu design, há reuniões com os stakeholders buscando eliminar incompatibilidades e buscar soluções com maior facilidade de execução, (GRANSBERG; WINDEL, 2008; DEL VECCHIO, 2011). Esse processo de análise também é recomendado para empreendimentos com características únicas, com grande chance de erros durante o processo construtivo (GIBSON JR et al., 1996). Também é 
SIBRAGEC - ELAGEC 2019 - de 23 a 25 de outubro - LONDRINA - PR

possível inserir conhecimentos advindos de uma avaliação pós-ocupação (GARSDEN, 1995) como facilidade de manutenção predial.

Outras pesquisas buscaram esclarecer a relação de construtibilidade com outras variáveis, sendo a principal a produtividade da mão de obra (JARKAS, 2010). Kifokeris e Xenidis (2017) apresentam ligações com diferentes áreas do conhecimento na construção civil (performance total da construção, gestão do conhecimento, análise de custo-benefício e outras).

A abordagem de "questões relativas à construtibilidade em diferentes sistemas prediais" inclui publicações que elucidam aspectos tecnológicos para algum sistema construtivo em específico, em busca de maior construtibilidade. Uma dessas publicações foi a de Navon, Shapira e Shechori (2000), com aspectos relacionados às dimensões e posicionamento de armaduras longitudinais positivas e negativas em vigas de concreto armado.

Uma ferramenta útil ao processo de análise de construtibilidade é o uso de BIM ou outros recursos computacionais. Kannan e Santhi (2018) apontam que a análise poderia usar recursos de BIM 4D (três dimensões espaciais e outra de tempo) para antecipar processos construtivos por simulação do empreendimento, ou na utilização de um plugin que lê dados do modelo BIM.

Quanto à abordagem quantitativa da construtibilidade, na forma de indicadores, artigos internacionais demonstram o funcionamento dos sistemas estatais de Singapura, como se dá a pontuação e de que forma os mesmos foram produzidos. Há ainda algumas pesquisas que propõem os seus próprios modelos de medição quantitativa da construtibilidade como Kannan e Santhi (2018) ou Jarkas (2010) - ambos os autores dão enfoque ao serviço de produção de formas.

No Brasil, apenas a dissertação de Narloch (2015) buscou produzir um sistema de indicadores de construtibilidade, com foco em aspectos geométricos do edifício, considerando princípios como padronização e repetição. Não considerados na revisão integrativa, mas detectados na narrativa estão duas monografias que verificam os escores de edifícios nacionais em sistemas internacionais. Uma pesquisa em que se considere tanto geometria como aspectos qualitativos e dados de produtividade, visando indicadores de construtibilidade, é uma lacuna de pesquisa existente na bibliografia brasileira.

De forma a sintetizar o processo evolutivo exposto, a Figura 4 traz os principais aspectos sobre "construtibilidade" desde os anos 1980 até o ano de 2019: 
SIBRAGEC - ELAGEC 2019 - de 23 a 25 de outubro - LONDRINA - PR

\section{Figura 4 - Evolução do conhecimento em construtibilidade}

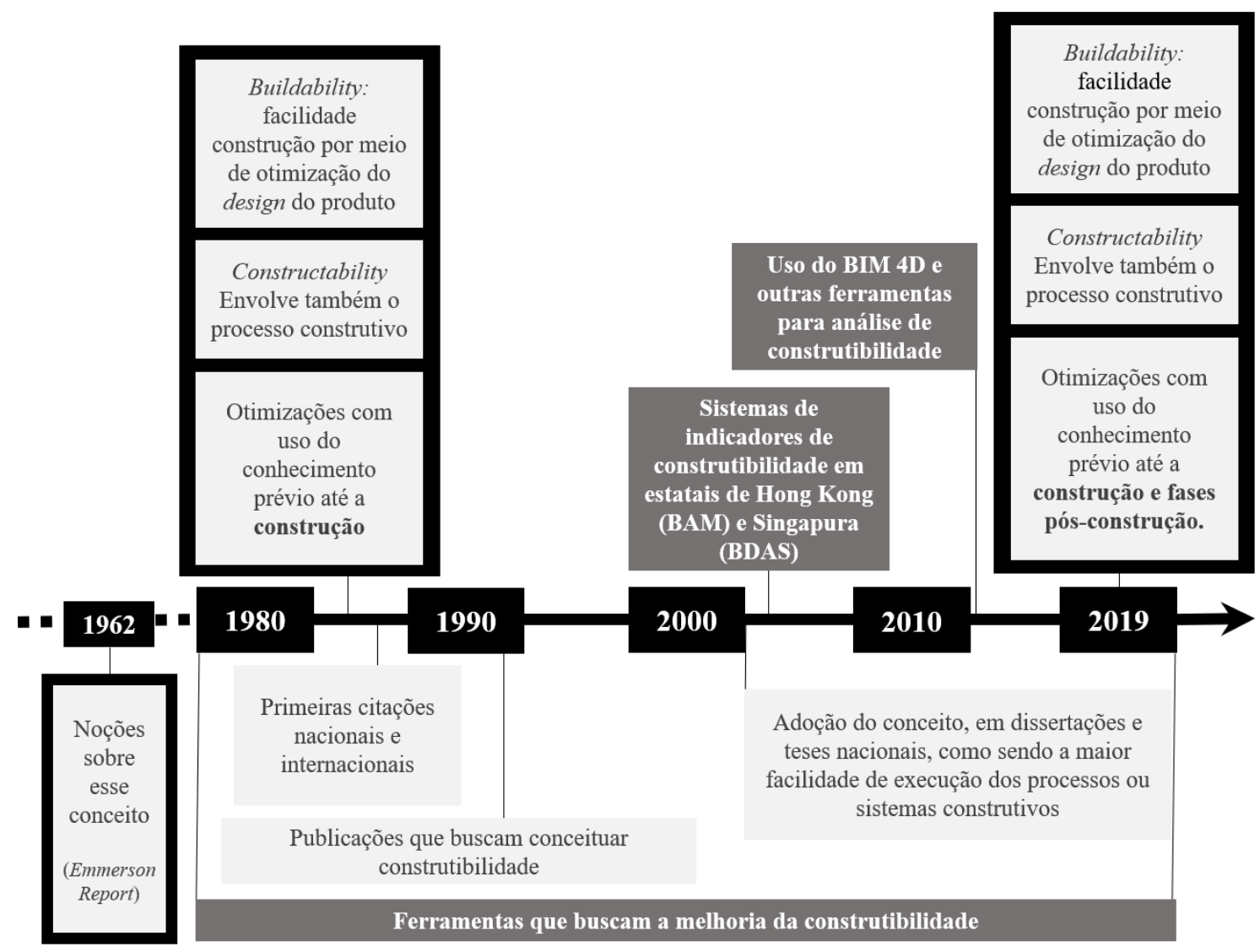

Fonte: os Autores (2019)

\section{CONCLUSÕES}

As pesquisas científicas relacionadas à construtibilidade buscaram, desde as primeiras definições de buildability e constructability até então, demonstrar de que maneira seria possível introduzi-la como filosofia nos empreendimentos de construção civil, com quais ferramentas e processos e os benefícios nessa introdução. Com diferentes abordagens, nota-se a evolução no conceito de construtibilidade na forma com que o mesmo se faz presente nas referências consideradas.

Não é mais desafio o entendimento do que seria construtibilidade, tanto que a mesma é utilizada de forma corrente em relatórios de pesquisa nacionais. Por outro lado, torna-se relevante a pesquisa sobre como melhorar a construtibilidade de edificações, incluindo ferramentas como BIM e outras que possam se incorporar ao mercado da construção, como indicadores de construtibilidade voltados à realidade brasileira.

Este artigo cumpre com o objetivo de traçar a evolução no conhecimento em construtibilidade. Espera-se que essa pesquisa sirva de base para a elaboração de um sistema de indicadores de construtibilidade a ser aplicado na realidade da construção civil brasileira, propiciando tanto a empresas públicas quanto privadas, a tomada de decisão quanto à escolha de projetos e sistemas construtivos. 
SIBRAGEC - ELAGEC 2019 - de 23 a 25 de outubro - LONDRINA - PR

\section{REFERÊNCIAS}

AMERICAN SOCIETY OF CIVIL ENGINEERS - Construction Management Committee of the ASCE Construction Division. Constructability and constructability programs: white paper. Journal of Construction Engineering and Management. v.117, n.1, p. 67-89, 1991.

Disponível em: https://ascelibrary.org/doi/abs/10.1061/\%28ASCE\%290733-

9364\%281991\%29117\%3A1\%2867\%29. Acesso em 01 fev. 2019.

ARAÚJO, Carlos Alberto. Bibliometria: evolução histórica e questões atuais. Em questão, v. 12, n. 1, 2006. Disponível em: http://seer.ufrgs.br/index.php/EmQuestao/article/view/16. Acesso em 13 mar. 2018.

BOTELHO, Louise Lira Roedel; CUNHA, Cristiano Castro de Almeida; MACEDO, Marcelo. O método da revisão integrativa nos estudos organizacionais. Gestão e Sociedade. v. 5, n. 11, p. 121-136, 2011. Disponível em:

https://www.gestaoesociedade.org/gestaoesociedade/article/download/1220/906. Acesso em: 16 ago. 2018.

CHIANG, Yat-Hung; CHAN, Edwin Hon-Wan; LOK, Lawrence Ka-Leung. Prefabrication and barriers to entry - a case study of public housing and institutional buildings in Hong Kong.

Habitat International. v. 30, p. 482-499, 2006. Disponível em:

https://www.sciencedirect.com/science/article/pii/S019739750500 0172. Acesso em: 02 fev. 2019.

DEL VECCHIO, Dennis. Absorber Implementation and Constructability Plan. Power

Engineering. p. 32-34, set. 2011. Disponível em: http://web-a-

ebscohost.ez46.periodicos.capes.gov.br/ehost/detail/detail?vid=2\&sid=34c79913-1064-4ee2-

81f8-e b949d7d0687\%40sessionmgr4008\&bdata=Jmxhbmc9cHQtYnIm

c210ZT1laG9zdC1 saXZl\#AN=66723251\&db=aph. Acesso em 13 fev. 2019.

GARSDEN, B.R. Postconstruction evaluation. Journal of Construction Engineering and Management. v.121, n. 1, p. 37-42, 1995. Disponível em:

https://ascelibrary.org/doi/abs/10.1061/\%28ASCE\%290733-

9364\%281995\%29121\%3A1\%2837\%29. Acesso em 04 fev. 2019.

GERTH, Robert; BOQVIST, Albert; BJELKEMYR, Marcus; LINDBERG, Bengt. Design for construction: utilizing production experiences in development. Construction Management and Economics. v. 31, n. 2, p. 135-150, 2013. Disponível em:

https://www.tandfonline.com/doi/abs/10.1080/01446193.2012.756 142. Acesso em: 04 fev. 2019.

GIBSON JR, George Edward. et al. Constructability in public sector. Journal of Construction Engineering and Management. v.122, n.3, p. 274-280, 1996. Disponível em:

https://ascelibrary.org/doi/abs/10.1061/\%28ASCE\%290733-

9364\%281996\%29122\%3A3\%28274\%29. Acesso em 13 fev. 2019.

GRANSBERG, Douglas D.; WINDEL, Elizabeth. Communicating Design Quality Requirements for Public Sector De-sign/Build Projects. Journal of Management in Engineering. v.24, n.2, p. 105-110, 2008. Disponível em: https://ascelibrary.org/doi/abs/10. 1061/\%28ASCE\%290742-597X\%282008\%2924\%3A2\%28105\% 29. Acesso em 13 fev. 2019.

JARKAS, Abdulaziz M. Analysis and Measurement of Buildability Factors Affecting Edge Formwork Labour Productivity. Journal of Engineering Science and Technology Review. v. 3, n. 1, p. 142-150, 2010. Disponível em: https://doaj.org/article/d31b9c768 2214eea9770a3e9a308e0df. Acesso em: 01 fev. 2019.

KANNAN, Ramesh; SANTHI, Helen. Automated constructability rating framework for concrete formwork systems. Asian Journal of Civil Engineering. n.19, p. 387-413, abr. 2018. Disponível em: https://link.springer.com/epdf/10.1007/s42107-018-0026-3?autho r_access_token=fNDz9MX-YpV-IVZKR55ob_e4RwlQNchNByi7w bcMAY6xeLhOtJVMh4M4cAHZOQSffFLiJmMzclFKVZhaZNWxRG7Z1dLxziKbSfJOIoHxz 
SIBRAGEC - ELAGEC 2019 - de 23 a 25 de outubro - LONDRINA - PR

G1Na54ZnkJkv5kKXSfgrIwvC4D3ghcbw pwO5rp36YrVOA\%3D\%3D. Acesso em: 01 fev. 2019.

KIFOKERIS, Dimosthenis; XENIDIS, Yannis. Constructability: Outline of Past, Present, and Future Research. Journal of Construction Engineeering and Management. v.143, n.8, 2017. Disponível em: https://ascelibrary.org/doi/abs/10.1061/\%28ASCE \%29CO.1943-7862.0001331. Acesso em: 04 fev. 2019.

LAM, Patrick T.I.; WONG, Franky W.H.; CHAN, Albert P.C. Contributions of designers to improving buildability and constructability. Design Studies. v. 27, n. 4, p. 457-479, 2006. Disponível em: https://www.sciencedirect.com/science/article/pii/S01 42694X05000918. Acesso em: 01 fev. 2019.

LAM, Patrick T.I.; WONG, Franky W.H.; CHAN, Albert P.C.; CHAN, Daniel W.M. Benchmarking Buildability Using the Buildability Assessment Model in Hong Kong. HKIE Transactions. v. 15, n. 1, p. 7-17, 2008. Disponível em: https://www.tandfonline.com/doi/abs/ 10.1080/1023697X.2008.10668104. Acesso em: 06 fev. 2019.

LIMA, Fernando Eliel de. Construções geométricas com o auxílio de régua e compasso do software Geogebra. 2018. Dissertação (Mestrado em Matemática) - Programa de Pós-graduação em Matemática, Universidade Estadual de Santa Cruz, Rio de Janeiro, 2018. Disponível em: https://sca.profmat-

bm.org.br/tcc_get.php?cpf $=08750173553 \& d=20190227151426 \& \mathrm{~h}=\mathrm{d} 6414 \mathrm{bc} 713 \mathrm{fc} 57 \mathrm{bab} 18 \mathrm{~d} 610$ 19e092aafc295593d Acesso em: 27 fev. 2019.

MACHADO JUNIOR, Celso Machado; SOUZA, Maria Tereza Saraiva de; PARIZOTTO, Iara Regina dos Santos; PALMISANO, Angelo. As Leis da Bibliometria em Diferentes Bases de Dados Científicos. Revista de Ciências da Administração, v. 18, n. 44, p. 111-123, 2016. Disponível em: http://www.spell.org.br/documentos/ver/40985/as-leis-da-bibliometria-emdiferentes-bases-de-dados-cientificos/i/pt-br. Acesso em 13 mar. 2018.

MELHADO, Sílvio Burattino. Qualidade do projeto na construção de edifícios: aplicação ao caso das empresas de incorporação e construção. 1994. Tese (Doutorado em Engenharia Civil) - Escola Politécnica da Universidade de São Paulo, São Paulo, 1994. Disponível em: http://www.infohab.org.br/acervos/sobreautor/codigoAutor/217/page/5/codigo_biblio/22547/cod/1. Acesso em: 13 fev. 2019.

NARLOCH, Tamyres Blenke. Modelo indicador da construtibilidade a partir da análise geométrica do projeto. 2015. Dissertação (Mestrado em Arquitetura e Urbanismo) - Programa de Pós-graduação em Arquitetura e Urbanismo, Universidade Federal de Santa Catarina, Florianópolis, 2015. Disponível em: https://repositorio.ufsc.br/handle/123456789/156886 Acesso em: 14 fev. 2019.

NAVON. Ronie; SHAPIRA, Aviad; SHECHORI, Y. Automated rebar constructability diagnosis. Journal of Construction Engineering and Management. v.126, n. 5, p. 389-397, 2000. Disponível em: https://ascelibrary.org/doi/abs/10.1061/\%28ASCE\%290733-9364 \%282000\%29126\%3A5\%28389\%29. Acesso em 13 fev. 2019.

POH, Paul S.H.; CHEN, Jundong. The Singapore Buildable Design Appraisal System: a preliminary review of the relationship between buildability, site productivity and cost.

Construction Management \& Economics. v. 16, n. 6, p 681-692, 1998. Disponível em: https://www.tandfonline.com/doi/abs/10.1080/014461998371971. Acesso em 02 fev. 2019.

SABBATINI, Fernando Henrique. Desenvolvimento de métodos, processos e sistemas construtivos - formulação e aplicação de uma metodologia. 1989. Tese (Doutorado em Engenharia Civil) - Escola Politécnica da USP, Universidade de São Paulo, São Paulo, 1989. Disponível em: http://www.teses.usp.br/teses/disponiveis/3/3146/tde-30082017-091328/ptbr.php. Acesso em: 01 fev. 2019.

TATUM, Clyde Bob. Building Better: Technical Support for Construction. Journal of Construction Engineering and Management. v.131, n.1, p. 23-32, 2005. Disponível em: 
SIBRAGEC - ELAGEC 2019 - de 23 a 25 de outubro - LONDRINA - PR

https://ascelibrary.org/doi/abs/ 10.1061/\%28ASCE\%290733-

9364\%282005\%29131\%3A1\%2823\%29. Acesso em 04 fev. 2019.

\section{AGRADECIMENTOS}

À CAPES, pelo apoio recebido no desenvolvimento dessa pesquisa. 